\title{
Cell stiffness and ROS level alterations in living neurons mediated by $\beta$-amyloid oligomers measured by scanning ion-conductance microscopy.
}

Oleg Suchalko ${ }^{1}$, Roman Timoshenko ${ }^{2}$, Alexander Vaneev ${ }^{3}$, Vasilii Kolmogorov ${ }^{1}$, Nikita Savin ${ }^{4}$, Natalia Klyachko $^{5}$, Evgeny Barykin ${ }^{6}$, Lyubov Gorbacheva ${ }^{7}$, Georgy Maksimov ${ }^{8}$, Sergei Kozin ${ }^{6}$, Alexander Erofeev $^{9}$, Yuri Korchev ${ }^{4}$, Pavel Novak ${ }^{10}$, Christopher Edwards ${ }^{11}$, Alexander Majouga ${ }^{12}$, Alexander Makarov ${ }^{6}$ and Peter Gorelkin ${ }^{9}$

${ }^{1}$ National University of Science and Technology "MISiS", Moscow, Russia, Moskva, Russia, ${ }^{2}$ National University of Science and Technology "MISIS", Moscow, Moskva, Russia, ${ }^{3}$ National University of Science and Technology "MISiS", Russia, "National University of Science and Technology "MISIS", Moscow, Russia, ${ }^{5}$ Lomonosov Moscow State University, Moscow, Russia, Russia, ${ }^{6}$ Engelhardt Institute of Molecular Biology, Russian Academy of Sciences, Moscow, Russia, United States, ${ }^{7}$ Russian National Research Medical University, Department of Fundamental and Applied Physiology, Moscow, Russia, United States, ${ }^{8}$ Lomonosov Moscow State University, Moscow, Russia, United States, ${ }^{9}$ National University of Science and Technology "MISiS", Moscow, Russia, Russia, ${ }^{10}$ National University of Science and Technology "MISiS", Moscow, Russia, United States, ${ }^{11}$ Imperial College London, London, United Kingdom, United Kingdom, ${ }^{12}$ D. Mendeleev University of Chemical Technology, Russia

Scanning ion-conductance microscopy (SICM) is a scanning probe microscopy technique with nanoscale resolution which allows for the surface topography investigation of nanoscale structures such as living cells in conditions close to physiological (Korchev et al., 1997). Apart from topography visualization, SICM can be used to estimate cell stiffness, which is based on elastic deformation due to colloidal pressure between nanopipette tip and cell surface, and to measure distribution of reactive oxygen species (ROS) concentration in cell.

In this work we have investigated alterations in neuron stiffness and ROS level mediated by $\beta$-amyloid oligomers. $\beta$-amyloids $(\mathrm{A} \beta)$ are specific molecules, which form amyloid plaques in human brain and are considered responsible for Alzheimer disease (AD) development (Holmes et al., 2008). Of all $\mathrm{A} \beta$ isoforms, $A \beta-42$ has been most studied and is also widely accepted as main neurotoxic form in $\mathrm{AD}$ (Arbor et al., 2016). The aggregation and accumulation of $\mathrm{A} \beta-42$ affect many cellular components, including plasma membrane and subcellular instances (Calamai et al., 2016) (Mokhtar et al., 2013). It is known that $A \beta-42$ increases ROS production, reduces mitochondrial membrane potential and leads to calcium dysregulation in neurons. The $\mathrm{A} \beta$ oligomers may interact with potential receptors, such as integrins, and trigger endocytosis (Verdier et al., 2004). Some studies also show that the cytoskeleton of the neuron may also be influenced by the $A \beta-42$ oligomer. The cytoskeleton is a fibrous network mainly composed of microtubules, microfilaments and intermediate filaments. A $\beta-42$ is reported to cause cytoskeleton modifications such as microtubule disassembly or actin polymerization, which results in spine and synapse degeneration in neurons (Ungureanu et al., 2016) (Qi Gao et al., 2019). These effects lead to the alterations in cell mechanical properties which can be estimated with SICM technique.

Using SICM we have estimated the Young's modulus and ROS concentration in control cells, after 4 hours and overnight treatment with $10 \mu \mathrm{M} A \beta-42$ oligomers in hippocampal neurons of rat. Our data show that incubation with $A \beta-42$ leads to drastic increase both in neuron 
stiffness and intercellular ROS level. It is known that incubation with $\mathrm{A} \beta$ oligomers results in significant increase in fibrillar actin (Mendoza-Naranjo et al., 2006), which may contribute to cell stiffness measured with SICM. Disrupted calcium homeostasis caused by A $\beta-42$ results in Rho GTPases activation, which are key regulators of F-actin polymerization (Bishop and Hall, 2000). At the same time, A $\beta-42$ induces mitochondrial fission in neurons and increases ROS production. Thus, using SICM technique we can confirm A $\beta-42$-induced alterations on stiffness and ROS level in hippocampal neurons.

The research was supported by the Russian Science Foundation Grant 20-14-00312.
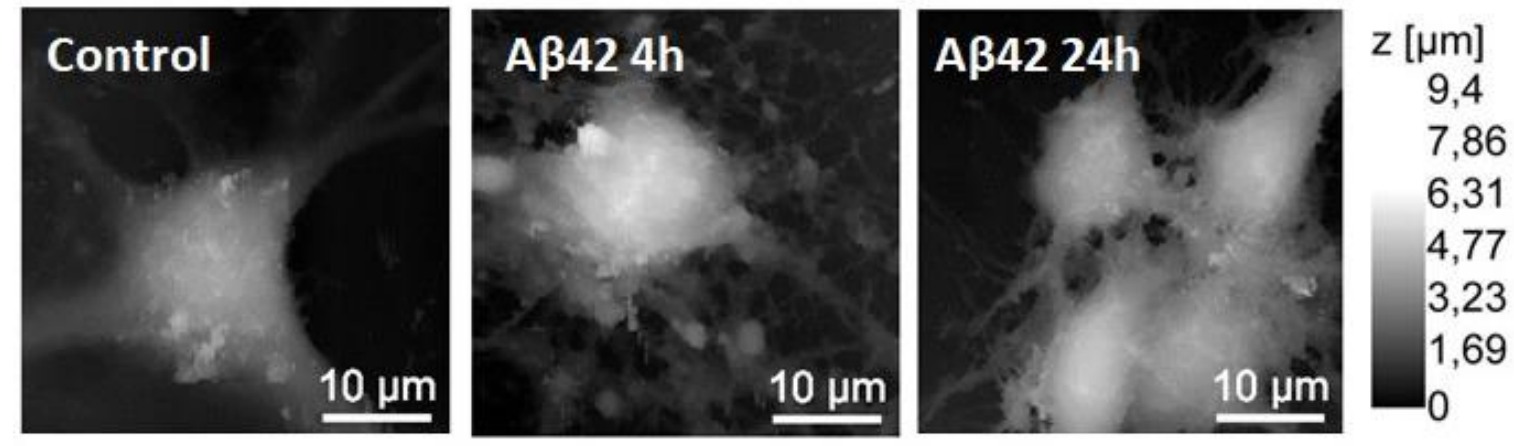

Figure 1. Topography alterations in hippocampal neurons of rat after treatment with A $\beta-42$ oligomers measured with SICM.
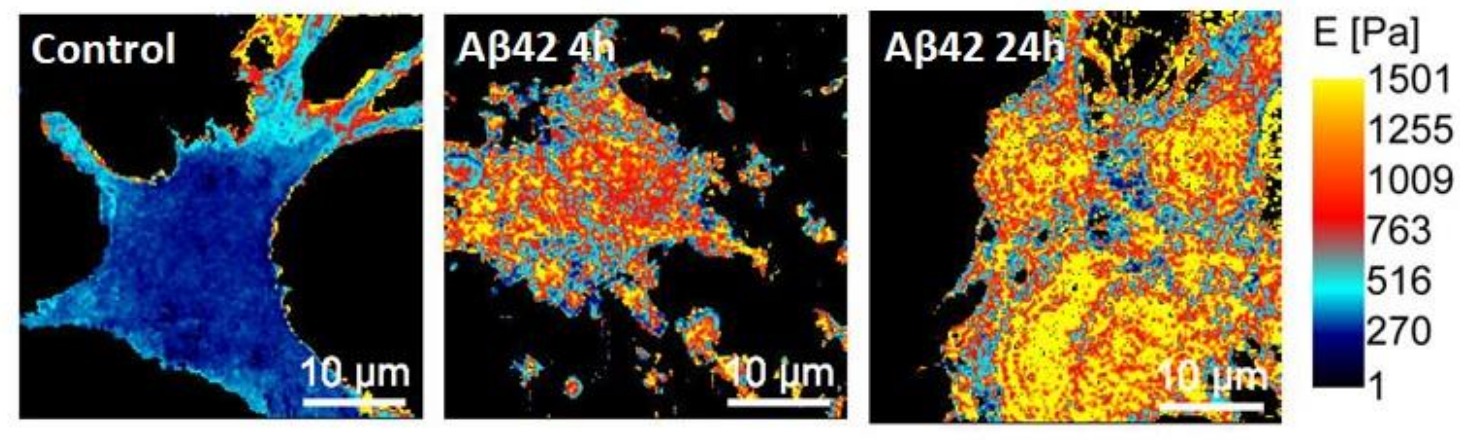

Figure 2. Stiffness alterations in hippocampal neurons of rat after treatment with A $\beta-42$ oligomers measured with SICM.

\section{References}

Arbor S.C., LaFontaine M., Cumbay M. (2016). Amyloid-beta Alzheimer targets-protein processing, lipid rafts, and amyloid-beta pores. Yale J Biol Med 89, 5-21.

Bishop, A. L. and Hall, A. (2000). Rho GTPases and their effector proteins. Biochem. J 348, 241-255.

Calamai, M., Evangelisti, E., Cascella, R., Parenti, N., Cecchi, C., Stefani, M., Pavone, F., (2016). Single molecule experiments emphasize GM1 as a key player of the different cytotoxicity of structurally distinct Ab1-42 oligomers. Biochim Biophys Acta 1858 (2), 386-392.

Holmes C., Boche D., Wilkinson D., Yadegarfar G., Hopkins V., Bayer A., Jones R.W., Bullock R., Love S., Neal J.W. (2008). Long-term effects of Abeta42 immunisation in Alzheimer's disease: Follow-up of arandomised, placebo-controlled phase I trial. Lancet 372, 216-223.

Korchev Y. E., Bashford C. L., Milovanovic M., Vodyanoy I., Lab M. J. (1997). Scanning ion conductance microscopy of living cells. Biophys. J. 73, 653-658 
Mokhtar, S.H., Bakhuraysah, M.M., Cram, D.S., Petratos, S. (2013). The beta-amyloid protein of Alzheimer's disease: communication breakdown by modifying the neuronal cytoskeleton. Int. J. Alzheimer's Dis.

Verdier, Y., Zarándi, M., Penke, B. (2004). Amyloid b-peptide interactions with neuronal and glial cell plasma membrane: Binding sites and implications for Alzheimer's disease. J. Pept. Sci. 10 (5), 229-248. Ungureanu, A., Benilova, I., Krylychkina, O., Braeken, D. (2016). Amyloid beta oligomers induce neuronal elasticity changes in age-dependent manner: a force spectroscopy study on living hippocampal neurons. Sci. Rep. 6, 1-13.

Mendoza-Naranjo A., Gonzalez-Billault C., Maccioni R.B. (2006). A $\beta 1-42$ stimulates actin polymerization in hippocampal neurons through Rac1 and Cdc42 Rho GTPases. Journal of Cell Science 120, 279-288. 